\title{
La docencia en el nivel de posgrado en el contexto de virtualización de emergencia. Aprendizajes y desafíos para el futuro en la experiencia de la Universidad Nacional de Tres de Febrero
}

Teaching at graduate level in the context of emergency virtualization. Learning and challenges for the future in the experience of the National University of Tres de Febrero

A docência no nível de pós-graduação no contexto de virtualização emergencial. Aprendizagem e desafios para o futuro na experiência da Universidad Nacional de Tres de Febrero

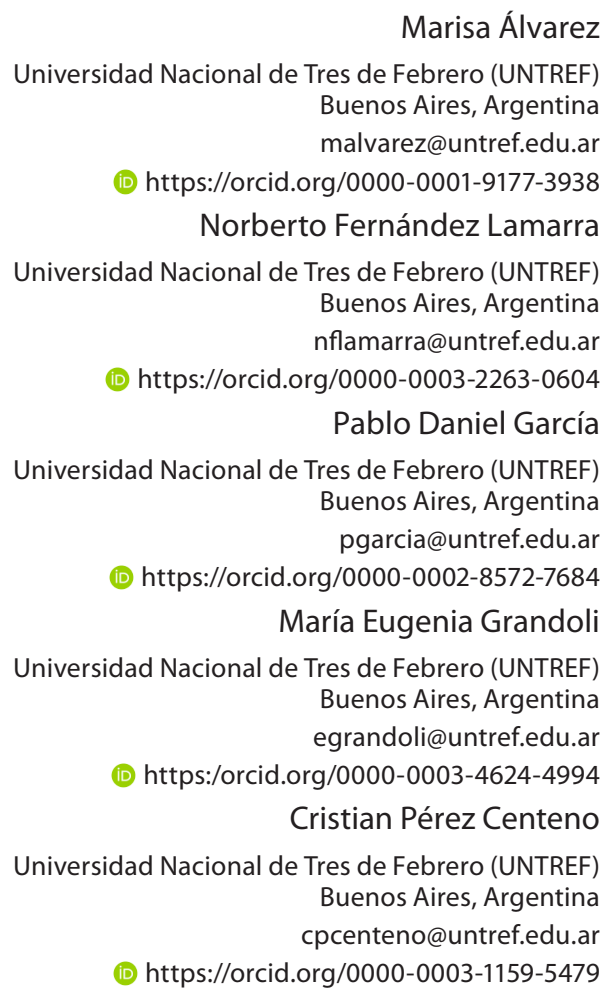

Recibido - Received - Recebido: 17 / 07 / 2020 Corregido - Revised - Revisado: 14 / 08 / 2020 Aceptado - Accepted - Aprovado: 03 / 09 / 2020

DOl: https://doi.org/10.22458/ie.v22iespecial.3153

URL: https://revistas.uned.ac.cr/index.php/innovaciones/article/view/3153 
Resumen: El trabajo presenta resultados parciales de un estudio de caso sobre la virtualización de la enseñanza "de emergencia", como respuesta a la interrupción forzosa del dictado de clases presenciales en el contexto de la actual pandemia por COVID-19. El caso analizado corresponde al nivel de posgrado de la Universidad Nacional de Tres de Febrero (UNTREF), en Argentina. La estrategia metodológica empleada fue de carácter cuantitativa y descriptiva. Consistió en la aplicación de un cuestionario autoadministrado en forma online al universo de docentes de posgrado que dictaron clases en el primer semestre de 2020, para conocer las percepciones y opiniones respecto del proceso de virtualización de la enseñanza.

El estudio muestra que, si bien la mayor parte de los docentes encuestados cuentan con una considerable experiencia de docencia en el posgrado, un tercio de ellos nunca había realizado tareas de docencia en entornos virtuales. Por otra parte, la mayoría de los docentes debió introducir cambios en su materia para adaptarla a las nuevas demandas de la virtualidad. Se destacan principalmente dos aprendizajes para el futuro desempeño docente, como frutos de la reciente experiencia de virtualización: el aprendizaje de soportes digitales novedosos que podrán emplearse en la enseñanza presencial, de manera de combinar el dictado regular con herramientas que provee la virtualidad, y la incorporación de herramientas tecnológicas que pueden sostenerse para mejorar la interacción con los estudiantes.

El artículo, finalmente, discute analíticamente las potencialidades, limitaciones y perspectivas futuras de la virtualización educativa en la emergencia.

Palabras clave: Enseñanza Superior, Educación a Distancia, Innovación Educacional, Universidad, Curso Postuniversitario, COVID-19

Abstract: The work presents partial results of a case study on the virtualization of "emergency" teaching, as a response to the forced interruption of face-to-face classes in the context of the current COVID-19 pandemic. The case analyzed corresponds to the graduate level of the National University of Tres de Febrero (UNTREF), in Argentina. The methodological strategy used was quantitative and descriptive. It consisted in the application of an online self-administered questionnaire to the universe of graduate teachers who taught classes in the first semester of 2020, to learn the perceptions and opinions regarding the teaching virtualization process.

The study shows that, although most of the teachers surveyed have considerable graduate level teaching experience, a third of them had never performed teaching tasks in virtual environments. On the other hand, most of the teachers had to introduce changes in their subject to adapt it to the new demands of virtuality. There are mainly two learnings for future teaching performance that stand out, resulting from the recent virtualization experience: the learning of new digital media that can be used in face-to-face teaching, in order to combine regular dictation with tools provided by virtuality, and the incorporation of technological tools that can be sustained to improve interaction with students. The article, in the end, analytically discusses the potentials, limitations and future perspectives of educational virtualization in emergencies.

Key Words: Higher education, distance education, educational innovation, university, post-university course, COVID-19

Resumo: $O$ documento apresenta os resultados parciais de um estudo de caso sobre a virtualização do ensino "emergencial", como resposta à interrupção forçada do ensino presencial no contexto da atual pandemia da COVID-19. O estudo de caso corresponde ao nível de pós-graduação da Universidad Nacional de Tres de Febrero (UNTREF), na Argentina.

A estratégia metodológica utilizada foi quantitativa e descritiva. Consistiu na aplicação de um questionário online autoadministrado ao universo de professores pós-graduados que ensinaram no primeiro semestre de 2020, a fim de conhecer as percepções e opiniões a respeito do processo de virtualização do ensino.

O estudo mostra que, embora a maioria dos professores pesquisados tenha considerável experiência de ensino na educação de pós-graduação, um terço deles nunca havia realizado tarefas de ensino em ambientes virtuais. Além disso, a maioria dos professores teve que fazer mudanças em seus temas para adaptá-los às novas exigências da virtualização. Há duas lições principais aprendidas para o desempenho futuro do ensino, como resultado da recente experiência de virtualização: o aprendizado de novas mídias digitais que podem ser usadas no ensino em sala de aula, de modo a combinar o ensino regular com ferramentas fornecidas pela virtualidade, e a incorporação de ferramentas tecnológicas que podem ser sustentadas para melhorar a interação com os alunos. Finalmente, o artigo discute analiticamente as potencialidades, limitações e perspectivas futuras da virtualização educacional no surgimento.

Palavras-chave: Ensino superior, educação à distância, inovação educacional, universidade, curso pósuniversitário, COVID-19 


\section{INTRODUCCIÓN}

A fin de enero de 2020, China informó la existencia de un nuevo virus de la familia de los coronavirus (SARS-CoV-2), el cual comenzó desde allí su expansión a escala mundial. La Organización Mundial de la Salud declaró formalmente al COVID-19 (Síndrome respiratorio agudo severo coronavirus 2) como pandemia el 24 de marzo de 2020 y los sistemas educativos a nivel planetario se vieron conmocionados. Una de las primeras decisiones de los gobiernos fue el cierre de las instituciones educativas de todos los niveles para garantizar condiciones de aislamiento social para frenar los contagios.

Este artículo propone un análisis de la respuesta que el profesorado de posgrado de una universidad argentina desarrolló ante la suspensión de emergencia de las clases presenciales para dar continuidad a su oferta académica.

En las últimas décadas, el nivel de posgrado en Argentina se ha expandido fuertemente, triplicando el número de ofertas de carreras y de estudiantes. Las modalidades de maestrías y especializaciones han sido dominantes en esta expansión (Barsky y Dávila, 2012). Según los últimos datos disponibles, existen 2977 carreras de posgrado, aunque solo 96 son "a distancia" (SPU, 2019), dando cuenta de la fuerte impronta presencial en la oferta académica de posgrado.

Uno de los problemas principales del posgrado refiere a la deserción y el desgranamiento de la matrícula. Así, la mejora de la retención y la graduación resulta estratégica para el nivel, que complementarán los avances logrados en la creación de nuevas carreras y la ampliación de la cobertura (Marquis, 2009). Por esto, la suspensión de las clases presenciales en el ciclo lectivo 2020 podría convertirse en un nuevo obstáculo para el sostenimiento de trayectorias de formación en el posgrado.

La emergencia de la COVID-19 llevó a la interrupción forzosa del dictado de clases presenciales y las instituciones educativas del nivel superior resolvieron la continuidad de acciones pedagógicas a través de medios digitales.

Este texto aborda el análisis de esta experiencia en el nivel de posgrado en la Universidad Nacional de Tres de Febrero (UNTREF), y busca responder las siguientes preguntas: ¿cómo ha sido la experiencia para las y los docentes involucrados en la virtualización "de emergencia" de su enseñanza?, y ¿qué aprendizajes e innovaciones pueden considerarse a partir del análisis de dicha experiencia en el futuro?

Este estudio, de carácter exploratorio y descriptivo, pretende hacer un análisis de las medidas adoptadas por el personal docente de posgrado de la UNTREF ante la interrupción de las clases presenciales y el impacto que tuvo en el desarrollo de los seminarios en curso. Se busca, además, indagar sobre las tensiones y los aprendizajes resultantes de la experiencia, así como las perspectivas de futuro respecto de la modalidad.

\section{MARCO CONCEPTUAL}

Este apartado se organiza en torno a tres ejes: el desarrollo del nivel de posgrado en Argentina, la innovación como objeto de investigación y el desarrollo de la educación a distancia y la virtualización educativa.

\section{El desarrollo del posgrado en Argentina}

La formación de posgrado argentina es "fundamentalmente profesionalista con sólo algunos bolsones de investigación científica y tecnológica, ubicados por lo general en áreas de las ciencias médico-biológicas y de las Ilamadas ciencias exactas" (Babini, Casalet y Oteiza, 1992: 286). La creación del Consejo Nacional de Investigaciones Científicas y Técnicas en 1958, produjo un nuevo impulso a la formación de 
posgrado (García y otros, 2016) bajo la forma honoríica del doctorado, evolucionando hacia estructuras ligadas a cátedras a la par de la consolidación disciplinar (Krotsch, 1996). Esta matriz maduró hacia su vinculación con actividades científicas y un modelo de grado continental europeo, mientras que, desde la década de 1960, el crecimiento de especializaciones y maestrías respondió a las dinámicas particulares de las disciplinas (García y otros, 2016). La irrupción de regímenes militares, la emigración de científicos y la prevalencia de políticas universitarias que no innovaron congelaron la situación existente (García y otros, 2016; Unzué, 2013). Investigaciones más recientes demostraron que los posgrados son el nivel de la enseñanza que más se expande, ligados al desarrollo profesional, científico y tecnológico del país y, no obstante ello, es el de menor intervención desde la política pública (Mazzola, 2015).

\section{El estudio de la innovación y la perspectiva de futuro}

El Núcleo Interdisciplinario de Formación y Estudios para el Desarrollo de la Educación (NIFEDE) de la UNTREF desarrolla, desde el año 2012, una línea de investigación en torno a la innovación en la educación superior cuyo objetivo es el de identificar y analizar experiencias de innovación institucional que hayan tenido incidencia favorable en las capacidades institucionales de formación, investigación y extensión. Los avances hasta el momento muestran que los procesos de innovación se originan en situaciones que combinan oportunidades sociohistóricas, la existencia de un grupo capaz de interpretar una necesidad colectiva y generar un proyecto, y la necesidad de resolver una situación problemática que afectaba a la organización en su conjunto. Por ello, la innovación produce un cambio profundo, que trastoca el sentido de un aspecto central de la institución. Se produce, entonces, una ruptura que moviliza la iniciativa individual hacia un "esfuerzo colectivo e institucional" que genera un cambio cultural en la organización que institucionaliza los cambios y los sostiene más allá de su propio contexto. Esto implica un doble esfuerzo: gestionar las resistencias a la innovación y dar visibilidad al cambio (Fernández Lamarra y otros, 2015).

El presente trabajo se enmarca en esta línea de investigación y busca analizar las experiencias desarrolladas por el profesorado en sus clases y las innovaciones desarrolladas en este marco, con perspectiva de futuro. Este enfoque permitirá elevarse más allá de las situaciones coyunturales para proyectarse en los tiempos que todo proceso y política educativa impone: el largo plazo. Esto requiere, a su vez, pensar en cambios que se están dando hoy, pero, sobre todo, discutir sobre la sociedad del futuro y sus características. Por ello, se adopta una intencionalidad prospectiva que permita advertir sobre las posibles consecuencias de las acciones presentes con vistas a futuros escenarios.

\section{La educación a distancia y la virtualización}

La educación a distancia en las universidades ha cobrado especial relevancia en los últimos años. Muchos autores han caracterizado esta modalidad (de Andraca, 2003; Filmus y otros, 2003; Olavarría y Carpio, 2004, entre otros). Beade y Díaz (2015) describen las características centrales del modelo contemporáneo de educación a distancia, a saber: separación espacial y/o temporal entre estudiantes y profesores y entre estudiantes, apoyado por medios didácticos diversos mediados por tecnología, autopreparación, autorregulación, trabajo independiente, necesidad de responsabilidad y motivación, actividad tutelar, entre otros.

García Aretio (2020) estudia la diversidad de significantes y significados asociados a la educación no presencial y define la educación a distancia como un

sistema tecnológico de comunicación bidireccional (multidireccional), que puede ser masivo, basado en la acción sistemática y conjunta de recursos didácticos y el apoyo de una organización y tutoría, que, separados físicamente de los estudiantes, propician en éstos un aprendizaje independiente y cooperativo (p. 23). 
Entre sus características centrales, menciona: la separación espacio-temporal del docente y los estudiantes en todo o parte del proceso de formación; la posibilidad del estudio independiente con control del tiempo, espacio, ritmo de estudio e itinerario formativo (incluyendo la evaluación); el soporte institucional en la planificación, diseño y producción de materiales; el seguimiento, orientación, evaluación y acreditación del proceso educativo; la comunicación e interacción sincrónica y asincrónica (según el grupo de estudiantes y el docente coincidan -o no- en el mismo espacio-tiempo).

Ahora bien, aunque la actitud hacia las tecnologías de la información y las comunicaciones (TICs) del profesorado y su formación tecnológica son claves para una buena experiencia de docencia en educación a distancia (Torres y otros, 2015), el verdadero desafío es la formación metodológica para el trabajo a distancia, la cual implica el desarrollo de competencias para el diseño de actividades y recursos de aprendizaje, así como para el uso de las herramientas didácticas idóneas (Cabero y Llorente, 2015).

En este trabajo se denomina "virtualización" o "modalidad virtual" a la estrategia de continuidad pedagógica a través de medios digitales realizada por la UNTREF ante la emergencia del COVID-19, que produjo la interrupción forzosa del dictado de clases presenciales. Se la distingue de la "educación a distancia", en tanto no responde plenamente a un modelo pedagógico de esa naturaleza. La virtualización de emergencia permitió la continuidad pedagógica a partir de un soporte on line (campus o aula virtual), pero a diferencia de la educación a distancia, dicho soporte ha sido utilizado en mayor medida como repositorio de documentos, también ha permitido la realización de actividades asincrónicas (favoreciendo el intercambio entre estudiantes y docentes) y, eventualmente, actividades sincrónicas. La virtualización refiere a lo que algunos autores denominan "aulas extendidas" o "blend learning" y que, estrictamente, consideran una propuesta de educación presencial extendida, a partir de la capitalización del uso de tecnologías (García Aretio, 2020).

\section{Sobre el caso de estudio: la UNTREF y las particularidades de su posgrado}

La UNTREF, creada en 1995, se encuentra ubicada en el Partido de Tres de Febrero de la Provincia de Buenos Aires, Argentina. Tiene sedes distribuidas geográficamente en las localidades de Sáenz Peña, Caseros, Villa Lynch y en Ciudad Jardín Lomas del Palomar. También tiene una sede específica de posgrados en la Ciudad Autónoma de Buenos Aires y más de noventa unidades de apoyo de educación a distancia distribuidas en todo el país.

Su creación se enmarcó en un plan de desarrollo de nuevas universidades que buscó disminuir la presión demográfica sobre las universidades tradicionales y atender demandas y necesidades de las comunidades donde se insertaron. Su proyecto institucional fue diseñado considerando la identidad local, el vínculo con la comunidad y la búsqueda de una mayor equidad en la enseñanza universitaria, facilitando el ingreso de estudiantes de la zona de influencia, en especial de los provenientes de sectores desprotegidos socialmente.

En particular, resulta importante destacar el desarrollo de un área de educación a distancia (UNTREF Virtual) creada en el año 2002 como unidad dependiente del rectorado y otra para el nivel de posgrados (presencial), cuyas primeras carreras se iniciaron en 1999.

La estrategia institucional consideró al desarrollo de Internet como una puerta de acceso a la educación a amplios sectores de la población. Entre sus propósitos se destaca la democratización y universalización del acceso a la universidad, brindando respuestas a las necesidades educativas y de formación de la comunidad con la ampliación de su ámbito geográfico de influencia (Guido y Versino, 2012).

En un primer momento, la oferta se dirigió al dictado de cursos de capacitación no académicos y luego se amplió la oferta a distancia a carreras de grado y posgrado. Actualmente, la Universidad ofrece, para el nivel de posgrado, cinco maestrías y cinco cursos de posgrado en modalidad a distancia. 
Según el Informe de Autoevaluación Institucional (UNTREF, 2018), en 2015, cursaron carreras virtuales 3.929 estudiantes y, en el período 2008-2015, habían cursado en esta modalidad 25.642. En 2018, UNTREF Virtual acreditó su Sistema de Educación a Distancia por parte de la Comisión Nacional de Evaluación y Acreditación Universitaria (CONEAU). Esta experiencia de dos décadas fue crucial en el momento de desarrollar con celeridad una propuesta pedagógica alternativa ante la imposibilidad de dictar clases presenciales.

Simultáneamente, en 1999, se creó el área de posgrado para ampliar la cobertura educativa en este nivel de formación, consolidando su proyecto institucional. Según el Informe de Autoevaluación Institucional (UNTREF, 2018), el crecimiento en el número de carreras que dicta y la matriculación de alumnos ha sido exponencial, incorporando una pluralidad de carreras de distintas ramas del saber, tales como gestión pública, derecho laboral, relaciones internacionales, periodismo, arte, cultura, educación, nuevas tecnologías, entre otras. Este desarrollo fue posible por la decisión y la voluntad institucional de promover nuevos espacios de formación de profesionales, actualización y articulación del saber en carreras destacadas por su originalidad y vanguardismo, diagramando su articulación con las carreras de grado y con las demandas sociales. Para el período 2008-2015, el número de estudiantes en el posgrado ascendió a 5410, con un significativo aumento progresivo en los últimos años. Sin considerar diversos cursos de posgrado que dicta en modalidad presencial y a distancia, en la actualidad la UNTREF ofrece 66 carreras de posgrado acreditadas por la CONEAU: 8 doctorados, 33 carreras de maestría y 25 especializaciones.

En otro orden, en el mes de marzo de 2020, el Decreto de Necesidad y Urgencia №260 estableció la Emergencia Sanitaria en el territorio nacional y, seguidamente, se anunció la suspensión por catorce días del dictado de clases presenciales en todos los niveles y modalidades para todo el país. A los pocos días, ante el aumento de casos positivos de COVID-19, se estableció el Aislamiento Social Preventivo y Obligatorio (ASPO) en todo el país para la totalidad de la población. El ASPO, sin embargo, fue prorrogado reiteradamente hasta el momento actual con diferentes modalidades en función de la evolución de la enfermedad en las distintas jurisdicciones, aunque persistiendo la imposibilidad de dictado de clases presenciales en todos los niveles de enseñanza.

Al momento de establecerse el ASPO, se lanzaba el año académico y solo unos pocos programas habían logrado iniciar la actividad (primer cuatrimestre). El mismo día que se decretó el aislamiento, la UNTREF informó la reprogramación de todas sus actividades, postergó el inicio del cuatrimestre hasta el 30 de marzo y suspendió los cursos ya iniciados. Asimismo, resolvió la virtualización del dictado de las asignaturas en los niveles de grado y posgrado.

Una de las primeras decisiones institucionales fue poner a disposición de las carreras la plataforma utilizada para el dictado de programas de formación a distancia. Esta opción no fue obligatoria, sino que las carreras y equipos docentes pudieron optar por su utilización, dada la provisoriedad del ASPO aunque, finalmente, alcanzó el primer cuatrimestre de manera total.

En este período se dictaron cursos correspondientes a 45 diplomaturas, especializaciones, maestrías (académicas y profesionales) y doctorados de todas las disciplinas. Esto puso en marcha el trabajo "virtualizado" en 120 asignaturas, alcanzando a 132 docentes.

\section{MATERIALES Y MÉTODOS}

En términos metodológicos, este estudio se basó en la autoadministración de una encuesta online (plataforma Survey-Monkey), anónima, aplicada a todos los docentes de posgrado que dictaron clases en el primer cuatrimestre de 2020 en la UNTREF. La participación en la encuesta no fue obligatoria y se enmarca en las regulaciones establecidas por el Comité de Ética de la UNTREF. 
Aunque la proporción de respondentes es alta, el estudio no pretende generalizar sus resultados, sino conocer la experiencia llevada a cabo y comprenderla desde la perspectiva de los docentes involucrados.

Las dimensiones de indagación a partir de las que se construyó el cuestionario fueron:

- modalidad de dictado de clase durante el período de vigencia del aislamiento,

- dispositivos utilizados y experiencia previa en el uso de los mismos,

- decisiones con respecto a la planificación del seminario y la selección de contenidos para el dictado de clases por virtualización de emergencia,

- experiencia de la virtualización lograda,

- evaluación de aspectos positivos y negativos de la experiencia desarrollada,

- innovaciones logradas y posibles incorporaciones a las futuras prácticas en el posgrado.

La encuesta, de carácter mixto, estuvo integrada por once preguntas cerradas (escala de Likert, de elección única y múltiple, etc.), once semi-abiertas y cinco abiertas. Se realizó una prueba piloto del instrumento.

El análisis de los resultados combina el análisis cuantitativo de las preguntas cerradas con el análisis cualitativo de las preguntas abiertas. Busca presentar, por un lado, las respuestas principales que brindan una orientación general respecto de lo sucedido; pero también, da cuenta de las opciones minoritarias que, en varias de las consultas, realizaron algunos docentes. En este caso permite ver disonancias o bien situaciones quizás poco frecuentes pero que pueden brindar elementos para pensar acerca del futuro de la virtualización educativa o de criterios que deben o deberían tenerse en cuenta.

Los datos obtenidos fueron procesados con el software IBM SPSS Statistics v.20.

\section{Resultados}

\section{Distribución de la muestra}

Del total de 132 docentes que virtualizaron sus actividades docentes, un total de 67 (50,7\%) han respondido la encuesta. De acuerdo con las respuestas de las Figura 1, Figura 2 y Figura 3, es posible establecer algunos rasgos centrales

Figura 1: Disciplina de desempeño

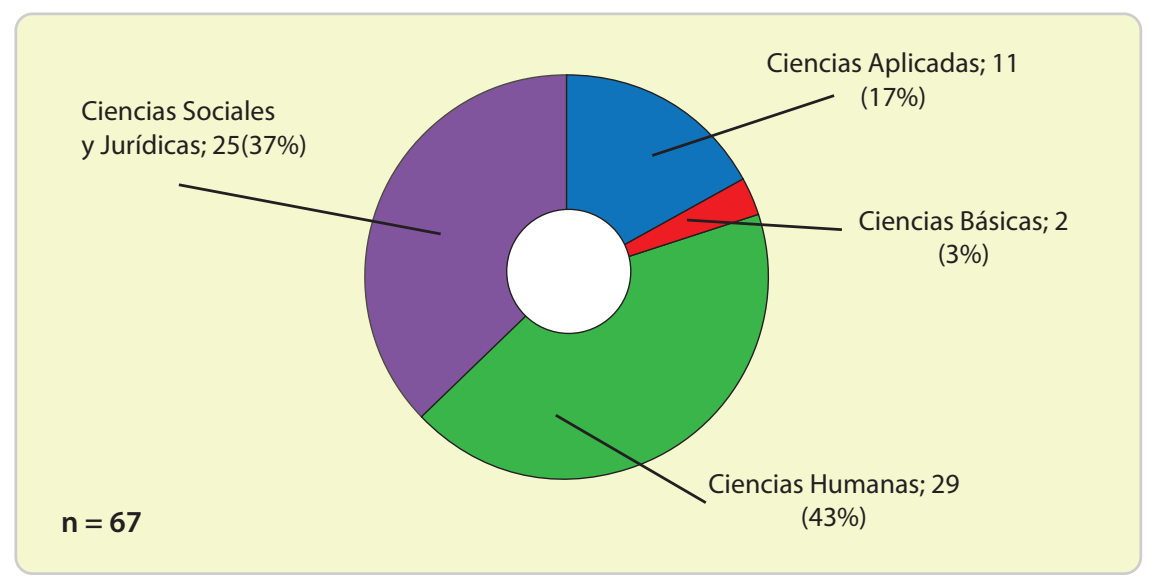

Fuente: Elaboración propia (2020) /

Nota: Se emplea la clasificación disciplinaria establecida por CONEAU. 
Figura 2: Experiencia en posgrados

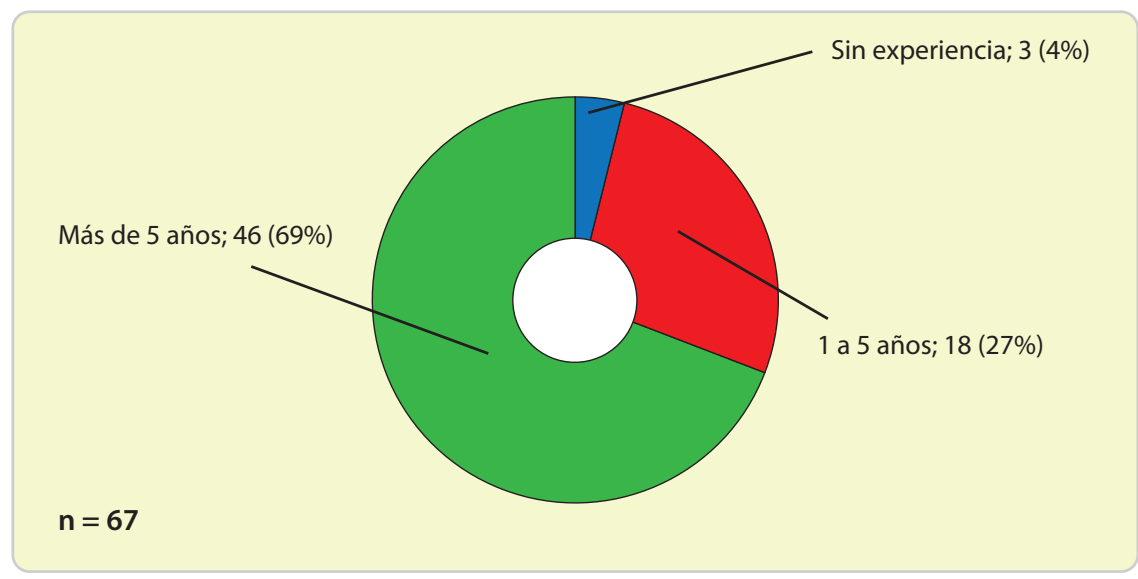

Fuente: Elaboración propia (2020)

Figura 3: Experiencia docente a distancia



Fuente: Elaboración propia (2020)

Se observa en la Tabla 1 que si bien una porción importante (43\%) de los académicos que tuvieron que virtualizar el dictado de su asignatura cuenta con experiencia de enseñanza en el nivel de posgrados y en entornos virtuales, un $37 \%$ de ese universo no cuenta con antecedentes suficientes para el manejo de este tipo de herramientas (menos de 5 años o sin experiencia).

\section{(ब) (1) $\Theta$}


TABLA 1

Área disciplinar de desempeño según experiencia de enseñanza en posgrados y entornos virtuales

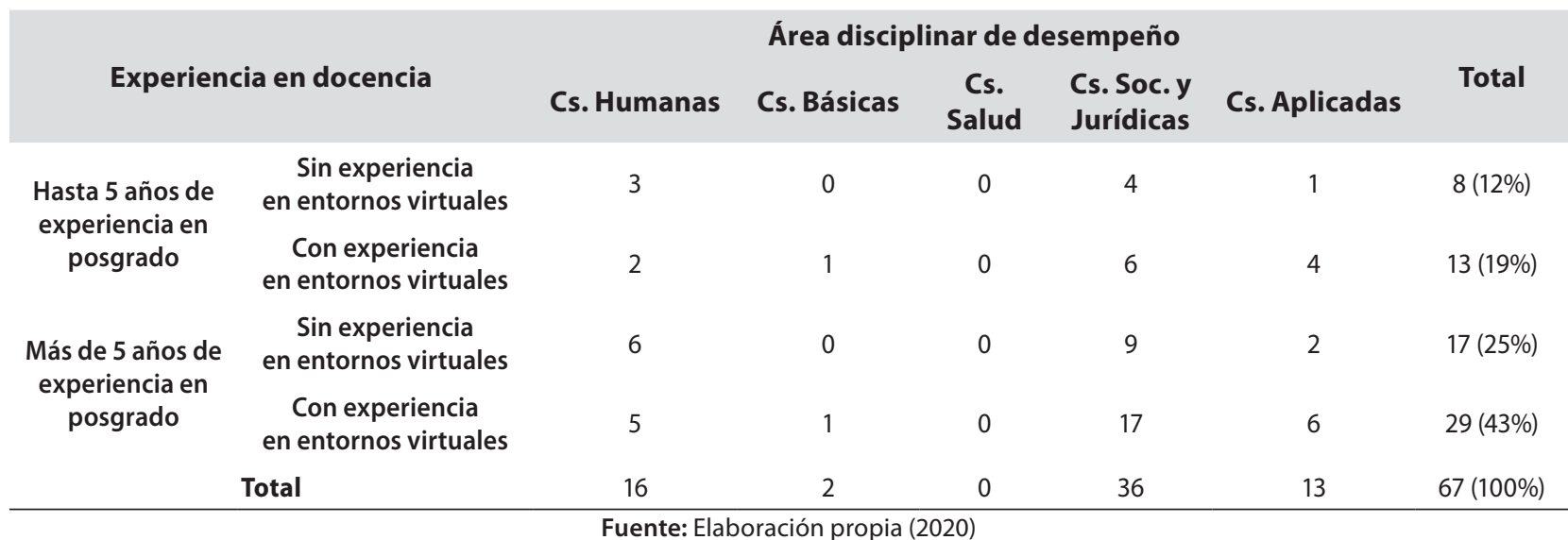

Así, si bien la mayor parte de los docentes cuentan con una considerable experiencia de docencia en el posgrado, un tercio de ellos nunca había realizado tareas de docencia en entornos virtuales. Este aspecto consideramos que resulta crucial al momento de tomar decisiones de emergencia frente a la imposibilidad de clases presenciales.

\section{Dispositivos y herramientas utilizadas}

Ante la suspensión de clases presenciales, hubo quienes optaron por continuar sus cursos de manera asincrónica, quienes optaron por un formato de clases sincrónico y quienes optaron por modalidades mixtas. En este sentido, es posible afirmar:

- $\quad$ La mayor parte de los docentes $(61,2 \%)$ eligió modalidades mixtas de enseñanza.

- Un 30\% del universo encuestado optó por dictar clases mediante la modalidad sincrónica.

- Apenas el 9\% del total eligió continuar el dictado de manera asincrónica.

TABLA 2

Modalidad de dictado elegida por los encuestados

\begin{tabular}{|c|c|c|}
\hline $\begin{array}{c}\text { Modalidad de dictado } \\
\text { elegida }\end{array}$ & Casos & $\begin{array}{l}\text { Porcentaje uso de mo- } \\
\text { dalidad de dictado }\end{array}$ \\
\hline Asincrónica & 6 & 8,9 \\
\hline Sincrónica & 20 & 29,9 \\
\hline Mixta & 41 & 61,2 \\
\hline Total & 67 & 100,0 \\
\hline
\end{tabular}

La modalidad elegida mantiene, en cada una de las disciplinas, el mismo criterio de preponderancia (la mayor parte de los docentes eligió una modalidad mixta, luego se ubican los que eligieron la modalidad sincrónica y, finalmente, es menor el grupo de docentes de cada grupo disciplinar que ha elegido solo la modalidad asincrónica para el dictado de clases virtuales).

Entre las reflexiones que realizan quienes han optado por la modalidad mixta, se destacan dos. En primer lugar, que primó la actividad sincrónica para el dictado de contenidos teóricos y esto se complementó con actividad asincrónica en foros. Por otro lado, varios casos destacan que las clases comenzaron 
mediante la modalidad asincrónica (por video pregrabado o clases escritas) y, a pedido de los estudiantes, fueron paulatinamente desarrollándose sincrónicamente. Al respecto, la Tabla 2 permite observar que, total o parcialmente, un $91 \%$ de los docentes tuvo clases "en directo" con los estudiantes para sostener la continuidad pedagógica durante el cuatrimestre.

Con respecto a las herramientas de las que se valieron los docentes para el dictado de las clases, 60 han manifestado que han usado algún tipo de videollamada (Zoom, Jitsi, Skype o similares), 49 docentes han usado la plataforma institucional, 6 han utilizado algún tipo de soporte web para grupos (Classroom, Teams, etc.) y 6 han elegido otras herramientas (YouTube, WhatsApp, Edmodo, mail, etc.).

Una hipótesis que puede sostenerse es que las decisiones pedagógicas tomadas a la hora de virtualizar las asignaturas se basan en la experiencia previa que cada docente tiene al respecto; es decir, podría presumirse un comportamiento diferenciado entre quienes poseían o no esa experiencia. Estadísticamente no fue posible probarlo debido a la baja cantidad de casos de uno y otro tipo. En el único caso donde sí fue posible realizarlo (uso de la plataforma institucional), el indicador no confirmó dicha asociación $(x 2, p=0,26)$, es decir, que no se encontró una relación en el hecho de tener experiencia como docente a distancia y haber elegido el campus de la UNTREF como soporte organizativo de la asignatura.

\section{Incidencia de la virtualización en la planificación de clases y la selección de contenidos}

\section{i. Cambio pedagógico y carga de trabajo}

La mayoría de los encuestados (84\%), independientemente de la disciplina, sostuvo que la virtualización implicó un cambio en sus tareas o en su rol como docente. De estos, un $84 \%$ señaló que la preparación de las clases le implicó más tiempo; esta proporción es menor entre los que no vieron modificado su rol (un 64\%). Los cambios identificados se relacionan con la preparación de las clases, de los materiales y bibliografía, de las actividades planteadas, pero también, con cambios en el vínculo con los estudiantes. Algunos docentes manifestaron una diferencia esencial entre las clases presenciales y virtuales y, en consecuencia, la necesidad de introducir cambios tanto en los contenidos como en las prácticas. Señalaron también la necesidad de adaptar las clases y el material utilizado al entorno virtual, preparar e incorporar más materiales, adaptar contenidos, reconvertir actividades y/o cambiar secuencias didácticas, lo que implicó, también, cambios en las distintas dimensiones pedagógicas.

Otro aspecto se refiere al vínculo con el estudiantado, que los interpela a modificar sus prácticas, tanto de planificación como de la forma en que los materiales son intervenidos para mejorar su comprensión a la distancia. Asimismo, señalaron los límites que la virtualidad impuso a la comunicación con los estudiantes. Los intercambios resultaron más pautados y restringidos, y, por lo tanto, el profesorado tuvo la necesidad de complementar la estrategia didáctica con otro tipo de actividades, tales como tutoría para los foros o la comunicación permanente a través de correo electrónico u otras herramientas de comunicación grupal. No obstante, las posibilidades que ofrece la comunicación asincrónica, el intercambio y el seguimiento personalizado, fueron valorados positivamente.

Todo esto les exigió desarrollar (aún con costos personales) más tareas para suplir la retroalimentación que supone la comunicación presencial y la disponibilidad de tiempo compartido para aclarar dudas y dar orientaciones al estudiantado. En general, los docentes se sienten interpelados a hacer un seguimiento más estrecho: hacer tutoría en la plataforma, intervenir en los foros, contestar consultas y dudas por escrito, promover más instancias de interacción. En algunos casos, se señala una mayor demanda de parte de los estudiantes.

Por otra parte, se revela cómo la tecnología predispuso (y es percibido de esta forma) un determinado tipo de comportamiento. La entrega de actividades parciales, correos electrónicos, devoluciones 
de actividades, promovieron una intervención docente diferenciada y una mayor interacción que durante las clases presenciales.

Sin duda, la referencia más reiterada fue la necesidad de una mayor dedicación horaria y disponibilidad para atender las demandas del estudiantado (consultas por mail o foros). Algunos docentes señalaron que debieron atender cuestiones personales de alumnos, tales como ansiedad, angustia o adaptación a nuevas prácticas.

A su vez, el profesorado admite cambios en sus prácticas: el reconocimiento de la capacidad de aprendizaje, la innovación en las actividades propuestas o cambios en sus propias actividades de formación (incluyendo el uso de plataformas, de nuevas herramientas, profundización del uso de las TICS, realizar capacitaciones ad hoc) o de otras actividades tales como la articulación o intercambio con otros docentes, ya sea de las propias cátedras como del intercambio institucional.

Resulta interesante la perspectiva de los docentes que, en general, no dieron cuenta de actividades que no pudieron realizar: solo en dos casos sostuvieron que afectó el desarrollo de actividades que se realizan en forma presencial.

ii. Los contenidos de las asignaturas.

Más del 92\% confirmó que las clases virtuales fueron iguales o más cortas que las presenciales; solo cinco casos afirmaron lo contrario. (Tabla 3).

En estos casos, los docentes dieron cuenta de la necesidad de cambiar, en parte, la dinámica de las clases presenciales, reordenar los contenidos y ofrecer mayores opciones de conectividad en días y horarios.

TABLA 3

Duración de las clases virtuales en comparación con las clases presenciales según modalidad de dictado utilizada

\begin{tabular}{ccccc}
$\begin{array}{c}\text { Modalidad de dictado } \\
\text { utilizada }\end{array}$ & \multicolumn{2}{c}{ Duración de las clases virtuales } & Total \\
Iguales & Más cortas & Más largas & \\
Asmbas & 15 & 23 & 3 & $41(61,2 \%)$ \\
Sincrónico & 2 & 4 & - & $6(9,0 \%)$ \\
Total & 8 & 10 & 2 & $20(29,8 \%)$ \\
\hline & $25(37,3 \%)$ & $37(55,2 \%)$ & $5(7,5 \%)$ & $67(100 \%)$ \\
\hline
\end{tabular}

Un docente da cuenta de que la mayor disponibilidad está asociada a aspectos emocionales, tales como

Intentar contagiar al grupo la pertenencia de grupo, estando a la distancia muchos se perdían en el inicio () Primero intenté conocer el grupo y las demandas (.) para acompañar el proceso y que no se sientan frustrados en la experiencia fui canalizando casi en el momento las consultas vía email, o llamadas por WhatsApp, no he tenido en cuenta horarios ni días, fueron todos los días iguales y me adapté a las necesidades de cada alumno.

Sin embargo, no hemos podido probar estadísticamente, dado que la cantidad de respuestas obtenidas en cada cruce de categorías de las variables analizadas impide estudiar su asociación, si la duración de las clases virtuales efectivamente se asocia a alguna otra característica, tal como la modalidad de dictado o la posibilidad de abordar los contenidos planificados (Tabla 4). 
TABLA 4

Abordaje de contenidos previstos según la duración de las clases virtuales

\begin{tabular}{ccccc} 
Extensión de las clases & Solo contenidos & & \\
en relación con las presenciales & $\begin{array}{c}\text { Contenidos abordados } \\
\text { mínimos }\end{array}$ & Muchos & Todos & Total \\
Más cortas & 1 & 21 & 15 & $37(55,2 \%)$ \\
Iguales & 1 & 10 & 14 & $25(37,3 \%)$ \\
Más largas & - & 2 & 3 & $5(7,5 \%)$ \\
Total & $2(3 \%)$ & $33(49,2 \%)$ & $32(47,8 \%)$ & $67(100 \%)$ \\
\hline
\end{tabular}

Fuente: Elaboración propia (2020)

Casi todos los docentes pudieron dictar los contenidos planificados totalmente $(47,8 \%)$ o en gran parte $(49,2 \%)$. Esto, probablemente se deba al hecho de que la adecuación a la modalidad virtual implicó, para una mayoría de docentes (55,2\%), la introducción de ajustes en los contenidos inmediatamente antes del inicio de las clases o que dichos cambios no alcanzaron al programa sino, más bien, a la forma en que fueron desarrollados o reorganizados.

Varios docentes dieron cuenta de un reemplazo de prácticas grupales por individuales, con devoluciones específicas, o algunas actividades pasaron directamente a ser virtuales, tales como asesoramiento, jurado de tesis, capacitación en nuevas plataformas, evaluación de proyectos de investigación.

Entre las dificultades que encontraron, los docentes mencionan que, en algunos casos, disminuyeron la cantidad de textos trabajados o que se restringieron algunos contenidos por las dificultades para trabajarlos en forma virtual. Consignaron que el aula virtual acotó las posibilidades de intercambio o presentó mayor dificultad que a nivel presencial. Los obstáculos más relevantes mencionados fueron "el menor tiempo disponible para desarrollar eficientemente las clases virtuales", "la falta de contacto visual con todos los alumnos", "limitaciones de recursos disponibles para algunos alumnos". El principal obstáculo -habiendo elegido la modalidad no sincrónica- fue la dificultad del intercambio en vivo con los alumnos.

Otros elementos explicativos frente a la dificultad de abordaje de los contenidos fue la precariedad del proceso de virtualización que, inicialmente, se presentó como provisorio y de corto plazo pero que terminó extendiéndose a la totalidad del dictado de las asignaturas, a la imposibilidad de optar por esta modalidad que la emergencia impuso y a la imprevisión que generó reorganizar la propuesta formativa de manera intempestiva ante el confinamiento obligatorio de la sociedad y el cierre de las universidades.

Respecto del grado de comprensión de los contenidos por parte de los estudiantes, la mitad de los encuestados no pudo dar cuenta de ello, dado que aún no había finalizado el cuatrimestre y no se llevaron a cabo las correspondientes evaluaciones. Quienes señalan aspectos de comprensión, en general, lo deducen de los intercambios en el aula virtual y de las actividades que se realizaron en el marco del dictado de la materia.

Algunos sugieren que sin "la interacción presencial y la posibilidad de hacer preguntas en el momento, de resolver dudas, y de hacer seguimiento presencial a los estudiantes" es más dificultoso o que, si "recibimos comentarios de que el cursado virtual fue difícil" o "no están preparados los hogares para funcionar como espacios para tomar clases", la comprensión no puede ser la misma que a nivel presencial. 
No obstante, otro grupo docente, en el extremo opuesto, reconoce que las herramientas digitales, con una adecuada planificación y uso, resultaron ser recursos positivos. La posibilidad de un trato personalizado e individualizado a partir del campus virtual favoreció la atención de inquietudes a intereses individuales. También se señaló que potenció la organización y el aprovechamiento de los tiempos para profundizar en la bibliografía y en los aspectos conceptuales y para mejorar la comunicación vía correos electrónicos. También se señaló que las clases virtuales más cortas ayudaron a la concentración y cumplimiento de los horarios.

\section{iii. El desarrollo de las clases y el vínculo con los estudiantes}

Si bien se desconoce si los equipos docentes ya utilizaban algunos de los recursos empleados en el formato virtual, la dinámica de las clases incluyó una gran variedad de estrategias y recursos. En general, se registra una combinación de clases sincrónicas (en las que se desarrollan los contenidos, que integran exposiciones con espacios de intercambios, consultas y dudas) con actividades a desarrollar por fuera de ese espacio.

Las plataformas virtuales permitieron la inclusión de clases grabadas, presentaciones comentadas, bibliografía, fichas temáticas, guías de lectura, pautas para el desarrollo de actividades y trabajos prácticos. Se señaló la incorporación de recursos on line utilizados como contenidos o como disparadores de actividades, incluyendo la producción especial de recursos para el dictado de las materias.

Un aspecto relevante señalado por el profesorado fue la interacción entre las clases asincrónicas, en los que se desarrollaron actividades prácticas, se elaboraron textos o consignas que luego fueron presentados en los encuentros sincrónicos. Así, la plataforma virtual parece haberse constituido en un soporte material para la organización del dictado de las asignaturas. Los testimonios del profesorado dan cuenta de distintos tipos de actividades desarrolladas en este marco: presentaciones temáticas, ejercitaciones, consultas, intercambios entre estudiantes, presentaciones en distintos formatos, entre otras.

En algunos casos se promovió el trabajo en grupos de estudiantes, a partir de la creación de subgrupos en la propia plataforma. También, algunos docentes reportaron el uso de diferentes herramientas o dinámicas a medida que fue avanzando el cuatrimestre, lo que muestra, por otro lado, cierta capacidad de reflexión y aprendizaje ante la situación extraordinaria de aislamiento.

A pesar de las dificultades señaladas y mayores demandas en la preparación de las clases y la disponibilidad para responder consultas de los estudiantes, el $97 \%$ de los docentes evaluó positivamente su desempeño en las clases virtuales (conforme 66\% y $32 \%$ muy conforme). En general, la conformidad respecto del desempeño tuvo como indicadores señalados por los docentes la alta asistencia y participación de estudiantes, la buena respuesta en la resolución de ejercicios y actividades, el cumplimiento con los contenidos previstos, la capacidad de contención y acompañamiento de los estudiantes y el compromiso que estos tuvieron.

Por otro lado, en relación con la dinámica, algunos docentes dieron cuenta de una mejor organización y aprovechamiento del tiempo, en particular a partir de una adecuada combinación entre clase sincrónica y foros.

Más allá de la conformidad general, las limitaciones que se mencionan ofrecen elementos para tener en cuenta hacia el futuro, especialmente, aspectos pedagógicos y formación específica de docentes en modalidad a distancia (tales como la capacidad para generar más espacios de participación e intercambio, estrategias especiales de desarrollo de contenidos adecuados a la modalidad digital o estrategias de construcción de contenidos virtuales). 
Por último, más del 90\% de los docentes consideró que la interacción con los estudiantes fue positiva, buena (43\%) o muy buena (48\%). Incluso, el 66\% señaló que incrementó la interacción con los estudiantes, que permitió una comunicación más personalizada con los estudiantes $(72 \%)$, la expresión de opiniones (68\%) y el trabajo colaborativo entre estos (66\%).

\section{DISCUSIÓN: POTENCIALIDADES, LIMITACIONES Y PERSPECTIVAS FUTURAS DE LA VIRTUALIZACIÓN EDUCATIVA EN LA EMERGENCIA}

La mayoría de los docentes encuestados debió introducir cambios en su materia para adaptarla a las nuevas demandas de la virtualidad. En líneas generales, dichos cambios han girado en torno a los contenidos del programa (priorización de unos sobre otros), la estrategia didáctica para desarrollarlos (incorporación de materiales audiovisuales, actividades prácticas), el soporte de la clase (uso de la plataforma institucional y de acceso gratuito para videoconferencias) y el modo de interacción con los estudiantes (mediado por foros, chats y correos electrónicos).

La evaluación, en cambio, prácticamente no sufrió modificaciones, lo que puede explicarse por la especificidad del nivel de posgrado. Aquí la evaluación generalmente se realiza mediante la entrega de producciones escritas, o productos técnicos que atienden una consigna particular, que debe entregarse al docente o a las autoridades de cada carrera en un cierto plazo.

A continuación, se realiza un balance de los aspectos positivos de la experiencia de virtualización de la enseñanza que atravesaron los docentes encuestados, así como sus limitaciones y aprendizajes para el futuro.

\section{Aspectos positivos de la virtualización}

Entre los más significativos se mencionan aquellos referidos a la relación pedagógica, como la mejora de la interacción docente-estudiantes, en tanto es más frecuente y personalizada. Incluso, se señala el mayor compromiso de los estudiantes para con el sostenimiento de la cursada.

También se mencionan aspectos positivos en torno a lo didáctico, especialmente la facilidad de acceder a recursos audiovisuales y digitales durante las clases, la posibilidad de acceder a nuevas herramientas tecnológicas, la preparación de nuevos materiales de apoyo y una mayor oportunidad para incluir invitados en los encuentros sincrónicos.

Por último, se mencionan aspectos materiales que ofrecen ciertas ventajas, como la posibilidad de ampliar y mejorar el acceso de aquellos que viven lejos del lugar de cursada; incluso, se señala que la virtualidad mejora la asistencia y puntualidad de los estudiantes, permitiendo organizar mejor sus tiempos. También se valora el ahorro de tiempo y recursos de traslado, así como cierta comodidad que ofrece el desarrollo de clases en el hogar. En este punto, debería indagarse con mayor profundidad la referencia a la "comodidad" señalada, tanto en cuestiones materiales vinculadas a la conectividad y recursos disponibles como a cuestiones ambientales domésticas (dado que el confinamiento forzó a realizar estas actividades docentes en condiciones personales muy diversas, que incluyen la convivencia y el cuidado de menores, mayores adultos o personas que requieren atención personalizada sin poder contar -en ocasiones- con personal específico para hacerlo debido a las restricciones del ASPO). 


\section{Limitaciones de la virtualización}

Una primera limitación tiene que ver con el vínculo entre docente y estudiantes. Se señala que la interacción diferida (por ejemplo, a través de foros o correo electrónico) le quita dinamismo a la comunicación, mientras que la interacción directa del encuentro sincrónico resulta un tanto rígida, más aún si la clase está siendo grabada, ya que se pierde espontaneidad en las intervenciones y cuesta que los estudiantes se expresen y participen libremente. En la interacción sincrónica, además, aparece la dificultad de poder captar el grado de atención o entendimiento de los estudiantes. Por otro lado, se señala la falta de intimidad que suele darse en el encuentro presencial, en el sentido de establecer contacto visual con los estudiantes, conocerlos y vincularse afectivamente con ellos.

En definitiva, estas limitaciones derivan en una menor interacción del docente con los estudiantes y de los estudiantes entre sí, al tiempo que se experimenta una pérdida en la calidad de las interacciones.

Otro conjunto de limitaciones se refiere a cuestiones didácticas. Las principales tienen que ver con la dificultad para que los estudiantes presenten temas en las clases, trabajen en grupos y se discutan contenidos e ideas. Por otro lado, se señala la falta de "simplicidad" que aporta el ámbito presencial, de poder disponer de un pizarrón en donde realizar anotaciones. Es evidente que algunas de las cuestiones planteadas aquí expresan -más bien- la falta de adecuada formación para el desarrollo de clases virtuales, ya que muchas plataformas o aplicaciones permiten subsanar aspectos analizados como limitantes. En este punto se vuelve clave la necesidad de asegurar la capacitación de los docentes para virtualizar la enseñanza y, en ese sentido, es que algunos encuestados han valorado negativamente la falta de asesoramiento técnico y pedagógico institucional para el desarrollo de actividades a distancia.

Por último, se identifican una serie de limitaciones materiales. Principalmente, el problema de la deficiente conexión a Internet en nuestro país, que fluctúa permanentemente y dificulta en especial la realización de encuentros sincrónicos. Asimismo, la falta de equipamiento adecuado de los estudiantes para poder acceder a determinadas propuestas pedagógicas, la competencia por recursos tecnológicos en el hogar en un contexto de obligaciones superpuestas (trabajo, escolaridad y vida doméstica). La sobrecarga del trabajo docente también aparece con fuerza en las respuestas, en la medida en que la preparación de clases adaptadas a la situación de virtualidad demanda mayor tiempo, tanto en el desarrollo de nuevos materiales como para el contacto con los estudiantes (mediatizado a través de correos electrónicos y foros). Pese a haberse incrementado notablemente la labor docente, la remuneración no sufrió modificaciones.

\section{Lecciones para el futuro}

La mayoría de los docentes encuestados considera que este periodo de enseñanza virtual ha dejado aprendizajes para el futuro en su desempeño docente. Principalmente se mencionan dos aspectos. En primer lugar, que esta experiencia ha derivado en el aprendizaje de soportes novedosos que pueden emplearse en la enseñanza presencial, de manera de combinar el dictado regular con herramientas que provee la virtualidad. En segundo lugar, que la experiencia ha habilitado la incorporación de herramientas tecnológicas que pueden sostenerse para mejorar la interacción con los estudiantes, asincrónicas como el uso de foros, y sincrónicas, a través de plataformas que permiten la realización de videoconferencias.

Luego se expresan reflexiones ligadas a la mejor regulación del tiempo, el abordaje de contenidos, la interacción con los estudiantes (intercambio, seguimiento de trabajos), la diversificación de actividades de aprendizaje, la producción de nuevos materiales y el desarrollo de formas de evaluación más creativas. 
En este sentido, parece que una perspectiva de futuro plausible es el desarrollo de modelos mixtos de enseñanza que articule las ventajas de los formatos presenciales y virtuales, lo que podría redundar en una mejora de los procesos educativos junto con la posibilidad de una mayor adecuación a las diversidades de estudiantes, en particular, fortaleciendo el cursado de asignaturas y evitando el desgranamiento y potenciales deserciones, así como la atención de situaciones excepcionales. No obstante, se advierte que este aporte no sobrevendrá por el mero uso de algunos dispositivos tecnológicos, sino por la implementación de una estrategia integral que lo sostenga.

La incorporación del uso sistemático de la plataforma virtual de enseñanza va en esa dirección, aún con diversas mejoras que podría requerir. La complementariedad de las instancias no presenciales permitiría descongestionar el uso de determinadas instalaciones como los laboratorios.

La sistematización de la formación de docentes en la enseñanza en entornos virtuales parece crucial para mejorar la planificación y el desarrollo de los procesos pedagógicos sin dejarlo librado a la posibilidad, capacidad y actitud de cada docente.

Parecería necesario un profundo análisis respecto de la carga laboral que implica la modalidad y las condiciones materiales y laborales para llevarla a cabo y la inclusión de espacios pedagógicos de intercambio de experiencias, con el fin de mejorar las prácticas.

Para concluir, se destaca que el objetivo ha sido analizar un caso particular de virtualización de la enseñanza ante la emergencia de la pandemia por COVID-19 y su consecuente confinamiento social. En todo caso, su valor reside en su propia singularidad y en las lecciones que es posible extraer para el futuro y no en el grado de representatividad que pueda tener. Los resultados aquí presentados, además, son preliminares y se trata de una investigación en curso. Se buscará avanzar hacia abordajes cualitativos que permitan profundizar en el análisis del caso. La triangulación de fuentes será estratégica en este sentido: la voz de los coordinadores de carrera y, sobre todo, de los estudiantes, podrán aportar nuevas reflexiones y puntos de vista que enriquecerán el estudio. Así, será posible sumar conocimiento para la innovación en las prácticas pedagógicas y de gestión institucional.

Es de esperar que estas experiencias y reflexiones puedan rápidamente traducirse en acciones y políticas educativas que atiendan los aspectos más críticos de esta emergencia: el acrecentamiento de la desigualdad educativa y la puesta en riesgo del derecho a la educación. Esto, en especial, si la principal reacción de los sistemas educativos nacionales ante el aislamiento y distanciamiento social obligado por la pandemia ha sido el cierre de las escuelas, la interrupción de todo proceso presencial de formación y la virtualización de la educación como principal estrategia de continuidad pedagógica y, además, si la responsabilidad central de este proceso ha recaído en el colectivo docente en las condiciones que tuvieran al momento del cambio de formato educativo (conocimientos, experiencia, grado de conectividad, recursos tecnológicos, condiciones personales y ambientales de trabajo en el hogar, etc.).

\section{REFERENCIAS}

Babini, D.; Casalet, M. y Oteiza, E. (1992). Recursos humanos en ciencia y tecnología. Formación de investigadores. En, Oteiza, E. (Dir.) La política de investigación científica y tecnológica argentina. Historia y perspectivas. Buenos Aires: CEAL.

Barsky, O. y Dávila, M. (2012). El sistema de posgrados en la Argentina: tendencias y problemas actuales. Revista Argentina de Educación Superior, Año 4, Número 5, octubre 2012.

Beade, L. y Díaz, T. (2015). Formación a distancia en el Doctorado en Ciencias de la Educación en Pinar del Río. Ponencia presentada en el evento “Universidad 2016". Pinar del Río, 27 junio. 
Cabero Almenara, J.; Llorente Cejudo, M. (2015). Tecnologías de la Información y la Comunicación (TIC): escenarios formativos y teorías del aprendizaje en Revista Lasallista de Investigación, vol. 12, núm. 2, 2015, pp. 186-193

de Andraca, A. M. (2003). Buenas prácticas para mejorar la educación en América Latina. Programa de Promoción de la Reforma Educativa en América Latina y el Caribe. Santiago: Editorial San Marino.

Fernández Lamarra, N. (Comp.), Aiello, M., Álvarez, M., Fernández L., García, P., Grandoli, M.E., Ickowiczz, M., Paoloni, P. y Perez Centeno, C. (2015). La innovación en las universidades nacionales. Aspectos endógenos que inciden en su surgimiento y desarrollo. Sáenz Peña: UNTREF.

Filmus, D.; González Pérez, O; Dias Pinto M.; Alvariño C., Zúñiga M. y Jara I. (2003). Educación y Nuevas Tecnologías. Experiencias en América Latina. Buenos Aires: IIPE-UNESCO.

García Aretio, L. (2020). Bosque semántico: ¿educación/enseñanza/aprendizaje a distancia, virtual, en línea, digital, eLearning...? Revista Iberoamericana de Educación a Distancia, 23(1), pp. 09-28. doi: http://dx.doi.org/10.5944/ ried.23.1.25495

García, L.; Cristaldo de Benítez, M; Di Marco C., Rodrigues Filho, J. y Zelaya M. (2016). Situación actual del posgrado en Argentina, Brasil y Paraguay: carreras, estudiantes y egresados. En: Lamfri, N. (Coord.) y otros (2016). Los posgrados en Argentina, Brasil y Paraguay: aproximaciones comparadas en contextos de evaluación de la calidad de la educación superior. Córdoba: Encuentro Grupo Editor.

Guido, L. y Versino, M. (2012). La educación virtual en las universidades argentinas. Buenos Aires: IEC-CONADU.

IESALC. (2020) COVID-19 y educación superior: De los efectos inmediatos al día después. Análisis de impactos, respuestas políticas y recomendaciones. Caracas: UNESCO.

Krotsch, P. (1996). El posgrado en la Argentina: una historia de discontinuidad y fragmentación. Pensamiento Universitario, № 4/5.

Marquis, C. (2009). Posgrados y Políticas Universitarias. Consideraciones sobre el caso argentino. Revista Argentina de Educación Superior, Año 1, Número 1, noviembre 2009.

Mazzola, C. (2015). La universidad de los gobiernos Kirchner. En: Pinto de Almeida y Otros (2015). Educação superior iberoamericana: uma análise para além das perspectivas mercadológicas da produção de conhecimento. Buenos Aires: CLACSO.

Olavarría, M. y Carpio, C. (2004). Educación a distancia y universidades. La experiencia de la GDLN. Ponencia presentada en el Congreso Virtual Educa Barcelona 2004.

Orozco, J. (2013). La Virtualidad en Educación Superior: Una Perspectiva. XIV Encuentro Internacional Virtual Educa Colombia 2013. Recuperado de http://www.virtualeduca.info/ponencias2013/171/ LavirtualidadenESUnaperspectivaJairoL.pdf

Secretaría de Políticas Universitarias (SPU) (2020). Síntesis de Información Estadísticas Universitarias 2018-2019. Buenos Aires: Ministerio de Educación.

Torres Gastelú, C.A., Lagunes Domínguez, A., Flórez García, M.A., Kiss, G. \& Alejandre Espinoza, A.R. (2015). Student's Perception about Online Interaction, Access and Publishing Content for Academic Use. The turkish online journal of educational technology, 14(3), 138-144.

Universidad Nacional de Tres de Febrero (2018). Informe de Autoevaluación institucional. Sáenz Peña (Argentina): UNTREF.

Unzué, M. (2013). Autonomía, evaluación y políticas públicas. Tendencias y límites en los sistemas universitarios de Argentina y Brasil. En Unzué, M. y Emiliozzi, S. (Comp.) Universidad y políticas públicas ¿en busca del tiempo perdido? Argentina y Brasil en perspectiva comparada Buenos Aires: Imago Mundi. 